\title{
Unterscheidung von bitteren Mandeln und Pfirsichkernen.
}

Von Professor Wittmack in Berlin.

Vortragender hat versucht, für die häufig beobachtete Verfälschung von bitteren Mandeln mit Pfirsichkernen (Prunus nana) den Nachweis durch anatomische Merkmale zu erbringen, was ihm auch gelungen ist. Nach diesen Untersuchungen sind die auf der Samenschale aufsitzenden und leicht abreibbaren Steinzellen bei den bitteren Mandeln stärker verdickt als bei dem Verfälschungsmittel. Weiter wurden noch Verfälschungen von bitteren Mandeln mit den Kernen von Pflaumen und Aprikosen beobachtet.

Als bestes Unterscheidungsmittel empfiehlt Vortragender den Geschmack herauszuziehen, der bei den bitteren Mandeln in Gegensatz zu den genannten Verfälschungsmitteln rein bitter und nicht unangenehm sei.

Anschliessend berichtet Professor Schär-Strassburg, dass in seinem Institut eine grössere Arbeit im Gange sei, welche bereits zu dem Ergebniss geführt habe, dass die in den verschiedenen Amygdaleen vorkommenden und bisher unter dem Namen Amygdalin zusammengefassten Glykoside verschiedene Körper seien. K. Lendrich.

\section{Referate.}

\section{Bier.}

William Frew: Die Bestimmung des Werthes der Brau- und BrennereiGerste. - Journ. Soc. Chem. Industr., 1901, 20, 221-223.

Der Verf. erwähnt, dass die Laboratoriumsuntersuchung der für technische Zwecke der Brauerei und Brennerei dienenden Gerste zu deren Bewerthung ebensowenig befriedigen kann wie die Beurtheilung nach äusseren Merkmalen. Er giebt dann die Methoden der Untersuchung im Laboratorium an. Von den einzelnen Bestimmungen werde der Keimversuch am häufigsten ansgeführt, dieser, sowie die mechanische Analyse, wie 1000 Körner-Gewicht, hl-Gewicht, Gestaltung, Reinheit etc., geben gute Anhaltspunkte zur Beurtheilung der Gerste für Mälzungszwecke. Verf. hat sich auch davon überzeugt, wie werthvoll für den Betrieb die Ausführung einer Mälzung in Laboratorium im kleinen Maassstabe und darauffolgende Darrung sein kann. Eine derartige Untersuchungsreihe mit verschiedenen Gersten zeigt, in welchem Zusammenhange die bei der Untersuchung der Gerste gewonnenen Werthe mit dem Ergebnisse der Mälzung und Darrung sich befinden können, insbesondere welche Bedeutung der jeweilige Stickstoffgehalt habe. Ferner wird betont, dass die Ergebnisse der Laboratoriumsmälzung mit denjenigen der Praxis sehr gut übereinstimmend waren, soweit dies durch die unvermeidlichen verschiedenen Einflüsse möglich erscheinen kann. Die aus 7 verschiedenen Gersten erhaltenen Ergebnisse waren folgende:

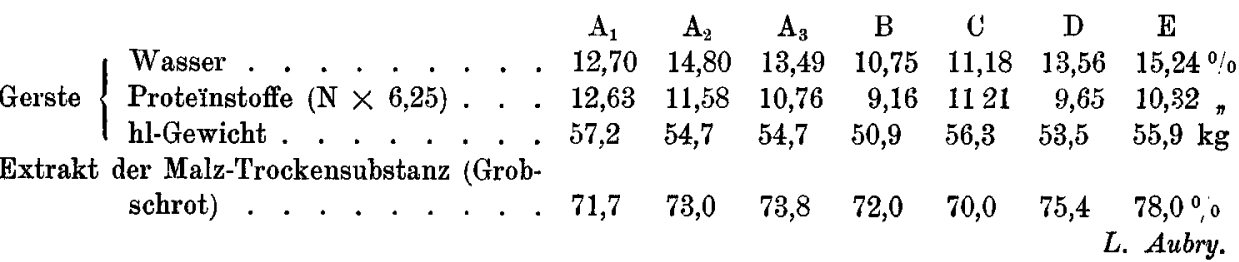


Moritz: Ueber den Einfluss des Trocknens der Gerste bei hoher Temperatur auf den Mälzungsprocess und die Malzbeschaffenheit. - Brew. Trad. Rer. 1900; Wochenschr. Brauerei 1901, 18, 2.

Ein grösserer Posten Gerste wurde in (lrei gleiche Theile getheilt, der eine Haufen wurde direkt vermälzt, der zweite zuvor bei $30-35^{0} \mathrm{R}$. und der dritte bei etwa $60^{\circ}$ R. 20-24 Stunden getrocknet. Der,Wassergehalt der Gersten war alsdann ungetrocknet $13,4 \%$, bei $30^{\circ} \mathrm{R}$. getrocknet $11,95 \%$ und bei $60^{0} \mathrm{R}$. getrocknet $11,3 \% \%$. Der Diastasegehalt der Gerste wurde nicht beeinflusst. Malz von obigem Wassergehalt würde entschieden, unter denselben Bedingungen getrocknet, einen Rückgang an diastatischer Kraft erleiden. Die Zusammensetzung der unter ganz gleichen V̀erhältnissen hergestellten Malze war nahezu dieselbe. Beide Malze aus den getrockneten Gersten waren heller in der Farbe. Aus den Versuchen kann zum mindesten der Schluss gezogen werden, dass das Trocknen der Gerste bei $60^{\circ} \mathrm{R}$. diese nicht schädigt. Auch vermuthet der Verf., dass durch das Trocknen der Gerste das Wachsthum auf der Tenne besonders bei zu hitzig und schnell wachsenden Gersten etwas zurückgehalten werde.

J. Brand.

E. Ehrich: Befindet sich im Malze ein eiweisslösendes Enzym? Bierbrauer 1901, 4.

Anknüpfend an die Arbeit Loe's (Diese Zeitschr. 1899, 2, 817), nach welcher ein eiweisslösendes Enzym im Malze nicht vorhanden ist, bringt Verf. einige Versuche über die Löslichkeit des Gersten- und Malzeiweisses zur Mittheilung. Aus seinen Versuchen über die Löslichkeit der Eiweissstoffe der Gerste zieht er den Schluss, dass zur lixtraktion der löslichen Stickstoffverbindungen eine zweistündige Maischdauer genügt, ferner, dass bei seinen Versuchen mit Gerste eine Enzymwirkung nicht wabrgenommen werden konnte. Dass bei der Extraktionstemperatur von $50^{\circ} \mathrm{C}$. wesentlich mehr Stickstoffsubstanzen als bei Zimmertemperatur löslich sind, führt Verf. nicht auf eine Enzymwirkung, sondern auf leichtere Löslichkeit dieser Substanzen in heissem Wasser zurück. Aus weiteren Maischversuchen bei $45^{\circ} \mathrm{C}$. und verschieden langer Zeit mit schlecht und normal gewachsenen Malzen, wobei bei längerem Maischen mehr Stickstoffsubstanzen in Lösung gehen, zieht Verf. den Schluss auf das Vorhandensein von eiweisslösenden Enzymen. Ein gleiches Ergebniss lieferte folgender Versuch: Gleiche Mengen Gerste und Malz wurden einerseits jedes für sich vermaischt und der Stickstoffgehalt der Würzen bestimmt, andererseits wurden die gleichen Mengen Gerste und Malz gemengt gemaischt und in gleicher Weise untersucht. Die Maische der Mischung von Gerste und Malz hatte bedeutend höheren Stickstoffgehalt, als die Summe des Stickstoffs aus der getrennt gemaischten Gerste und Malz ergab. Es war eine energische Wirkung des eiweisslösenden Enzyms des Malzes zu verzeichnen. Nach der Ansicht des Verf. dürfte auf Grund der mitgetheilten Versuche sowie anderer, die ein ähnliches Resultat ergeben haben, es nicht zu bezweifeln zein, dass im Malze ein eiweisslösendes Enzym vorhanden ist, ferner dass dieses Enzym in vielen Malzen eine sehr energische Wirksamkeit besitzt, in anderen Malzen dagegen weniger wirksam ist.

J. Brand.

Lawrence Briant: Die Bestimmung des nutzbaren Extraktes aus Malz. - Analyst 1901, 26, 2-8.

Verf. macht auf die grossen Differenzen aufmerksam, welche durch die verschiedenen Verfahren der Malzanalyse entstehen und glaubt, dass die beste Malz- 
analyse diejenige sei, welche sich dem Verfahren im Sudhause anpasse. Indem er auf das englische Brauverfahren der Infusion eingeht, zeigt er, wie die Extraktausbeute durch die Temperatur beeinflusst wird - [Eine in der deutschen Braulitteratur schon seit zwei Jahrzehnten genügend gewürdigte Thatsache - Ref.] - und geht alsdann auf das Malzuntersuchungsverfahren von Heron näher kritisch ein. Heron bringt $50 \mathrm{~g}$ Malz mit $400 \mathrm{ccm}$ Wasser bei $50^{\circ} \mathrm{C}$. zusammen und erwärmt die Maische im Wasserbad innerhalb einer Stunde auf $70^{\circ} \mathrm{C}$; ; dann wird in 15 Minuten auf $88^{\circ} \mathrm{C}$. erhitzt und alsdann sofort auf $15^{1} / 2^{0} \mathrm{C}$. abgeküblt und auf $515 \mathrm{ccm}$ verdünnt. Von der abfiltrirten Würze wird das specifische Gewicht genommen und daraus der Extrakt auf das Ganze berechnet unter der Annahme, dass die Extraktlösung genau ist. Diese Annahme wird mit Recht beanstandet und auf die Verbesserung durch Stern hingewiesen, der den Fehler durch das wechselnde Volumen der Treber mit einer Art von Zweifiltratsmethode zu beseitigen suchte, die aber nicht einwandfrei ist. Es wird alsdann folgendes Maischverfahren vorgeschlagen: Man maischt $50 \mathrm{~g}$ Malzschrot in einem Kolben mit $400 \mathrm{~cm}$ Wasser, das auf $71^{\circ} \mathrm{C}$. erwärmt wurde, verschliesst den Kolben lose, um die Verdunstung zu vermeiden, stellt den Kolben in ein Wasserbad von $65^{1 / 2^{0}} \mathrm{C}$. und hält diese Temperatur zwei Stunden an. Nach dieser Zeit wird auf $15^{1 / 2}{ }^{0} \mathrm{C}$. abgekühlt, mit noch $100 \mathrm{ccm}$ Wasser der gleichen Temperatur verdünnt, gut gemischt und filtrirt. Die Flüssigkeit dient zur specifischen Gewicbtsbestimmung. Die Berechnung wird an einem Beispiele gezeigt. Das spezifische Gewicht sei 1028 und wird das über 1000 gehende Gewicht durch den Flüs-igkeitsfaktor 3,86 dividirt, was 7,25 als das Gewicht der festen Substanz in 100 ecm der Würze ergiebt. Dieses mit 5 multiplicirt giebt 36,25 aus dem Malze aufgelöst. Da nun 15,9 Thle. feste Substanz (Zucker) einen Raum von 10 Thln. in Lösung einnehmen, so ergiebt sich, dass aus $50 \mathrm{~g} \mathrm{Malz} \frac{36,25 \times 10}{15,9}$ gelöst werden. Die Gesammtmaische beträgt daher 523 ccm. Es wird schliesslich zur Vereinfachung der Berechnung der Extraktionsbeute der Faktor 3,51 angegeben, mit welchem das Mehrgewicht von $1000 \mathrm{ccm}$ Würze multiplicirt, die Pfunde Extrakt per Quarter von 336 Pfund angiebt. Die Berechnung ist für englische Verhältnisse eingerichtet.

L. Aubry

E. Jalowetz: Vergleichende Untersuchungen überverschiedene Maischverfahren II. - Allgem. Zeitsch. Bierbrauerei u. Malzfabr. 1901, 28, 197.

Es werden die analytischen Ergebnisse der vom Verf, angestellten Vergleichssude zur Veröffentlichung gebracht. Ueber die daraus sich ergebenden Schlussfolgerungen wurde bereits früher (Diese Zeitschr. 1901, 4, 326) berichtet. Vergleichssude zwischen Drei- und Zweimaischverfahren im Jahre 1898/99 ergaben im Dextringehalte einen Unterschied von $0,36 \%$ zu Gunsten des Dreimaischbieres. Auch bei weiteren Versuchen, welche in der Dampfkochpfanne durchgeführt wurden, enthielt das Zweimaischbier um 0,3\% weniger Dextrin. Bei weiteren Vergleichsversuchen zwischen Dreimaisch-Verfahren und dem von $\mathrm{W}$ indisch lagen die Unterschiede in der Zusammensetzung innerhalb der Fehlergrenzen. Ein zweiter Parallelversuch mit dem gleichen Malze lieferte wieder vollkommen übereinstimmende Ergebnisse. Ein weiterer Parallelversuch, bei welchem eine andere Malzsorte verwendet wurde, lieferte dagegen wesentlich andere Ergebnisse und zeigte diewer Versuch, dass die Beeinflussung des Dextringehaltes im Biere in ungleich einfacherer Weise durch das Malz geschehen kann, als beim Maischprocesse; durch den letzteren lässt sich die Zusammensetzung der Würze nur innerhalb sehr eng gezogener Grenzen bei einem bestimmten Verfahren abändern, 
da beim Maischen in der Praxis nicht ausschliesslich die Kohlenhydrate zu berücksichtigen sind. In der Mälzerei scheint das angestrebte Ziel nicht schwer zu erreichen zu sein.

J. Brand.

A. Zeidler und M. Nauck: Ueber den Albumosengehalt von Würzen. Wochenschr. Brauerei 1901, 18, 101-107.

Vor einigen Jahren wies Windisch auf die Bedeutung der Albumosen im Biere hin, die einen entscheidenden Einfluss auf die Schaumhaltigkeit des Bieres haben sollen und stellte später nach dem Verfahren von A. Bömer aus Bier und Würze einen Eiweisskörper dar, der ein ausserordentliches Schaumbildungs- und Schaumhaltungsvermögen zeigte. Die Verfasser legten sich nun die Fragen vor:

1. Auf welche Weise erhält man albumosereichere Würze? und 2. Giebt einesolche Würze schaumhaltiges Bier? Zur Beantwortung der ersten Frage wurden eine Reihe von Versuchen in Mälzerei und Sudhaus angestellt, wobei die gleiche Gerste unter denselben Bedingungen gemälzt und die erhaltenen gleich gedarrten Malze in verschiedenen Sudwerken verarbeitet wurden. Im Sudhause wurde der Einfluss verschiedener Maischmethoden, Dauer und Energie des Hopfensudes, Einfluss der Temperatur des Anschwänzwassers und Dauer der Läuterzeit auf den Albumosengehalt der Würzen geprüft. Aus den Mälzereiversuchen ging klar hervor, dass Malze mit langem Gewächs und Blattkeim höheren Albumosengehalt ergeben und dass bei Verarbeitung von Gerste mit höherem Proteïngehalt der Albumosengehalt der Würzen erhöht wird. Es würde sich also die Vermuthung von $W$ indisch nicht bestätigen, dass kurzgewachsenes Malz wahrscheinlich mehr Eiweiss von Albumosencharakter besitzt als langgewachsenes. Fasst man die Ergebnisse aller Versuche zusammen, so wäre die Antwort auf die erste Frage folgende:

Man müsste bestrebt sein: 1. Ein Grünmalz mit möglichst langem Blatt- und Wurzelkeim dlarzustellen. 2. Die Höhe der Würze im Hopfenkessel zu beschränken. 3. Die Dauer des Hopfensudes zu kürzen. 4. Langsames Maischen und besonders niedere Maischtemperaturen zu vermeiden. 5. Die Temperatur des Anschwänzwassers, soweit es die Umstände erlauben, zu erhöhen. Ganz besonders wichtig erscheint die Arbeit auf der Tenne und in der Trommel.

Was die zweite Frage betrifft: Giebt eine solche (albumosenreichere) Würze schaumhaltigeres Bier? so musste dieselbe im vorliegenden Falle verneint werden. Es konnte bei Untersuchung der Biere (Flaschenbiere) kein Unterschied in der Schaumhaltigkeit bemerkt werden. Dafür wurde ein nicht minder wichtiges Resultat erzielt:

Alle Biere, die mehr Albumose enthielten, hatten einen ausgesprochen milderen runden Geschmack, der um so deutlicher hervortrat, je mehr Albumose vorhanden war. Unter $5 \%$ war der Unterschied schwerer zu erkennen.

Die Verff. glauben, dass dieses Ergebniss Grund genug giebt, Interesse für die Frage zu erwecken und lohnend genug ist, die Sache weiter zu verfolgen.

J. Brand.

N. van Laer: Ueber das Abkühlen, Filtriren und Karbonisiren von obergährigen Bieren. - Journ. Federat. Inst. Brew. 1900, 6, 439; Wochenschr. Brauerei 1901, 18, 70.

Das schnelle Abkühlen des Bieres durch Hindurchleiten durch gekühlte Kupferrohre hat sich aus verschiedenen Gründen nicht bewährt. Das langsame Abkühlen wird in der Weise bewirkt, dass das Bier nach 14-tägigem Lagem in einen kalten 
Raum verbracht wird, descen Temperatur unter $0^{0}$ gehalten wird. Hierbei scheiden sich langsam Hefe und andere Stoffe aus und setzen sich ab. Nach etwa einer Woche wird das fast blanke Bier filtrirt, was nun leicht von statten geht. Nach Beobachtungen des Verf.'s gehen beim Abkühlen und Filtriren des Bieres das feine Hopfenaroma, sowie auch Vollmundigkeit verloren. Ein auf diese Weise abgekühltes und filtrirtes Bier, das beim Füllen auf Flaschen noch karbonisirt wird, ist nach Ansicht des Verf.'s nicht mehr kälteempfindlich, es kann zu Eis gefrieren ohne Ausscheidungen oder einen Bodensatz zu geben. Biere mit ausgesprochenem Hopfencharakter, trocken gehopfte Biere, die längere Zeit lagern sollen, eignen sich für dieves Verfahren nicht. J. Brand.

John Ryder und Alfred Greenwood: Arsen im Bier. - Chen. News 1901, 83, 61 .

Zur Bestinmung des Arsens in Bier haben die Verf. folgendes Verfahren angewandt. 21 Bier (oder auch weniger, je nach Ausfall der qualitativen Prüfung) werden mit 3 bis $4 \mathrm{~g}$ blanken Kupferblechs in kleinen Stücken und 1/6 1 reiner Salzsäure von 1,16 spec. Gew. eine Stunde lang gekocht. Eine andere Probe Bier wird mit $1 g$ Kupferblech noch eine Viertelstunde länger gekocht und, wenn hierbei keine Flecken auf dem Kupferblech entstehen, bei Seite gestellt. Das Kupfer von beiden Proben wirl mit Wasser abgespült, in Salpetersüure gelöst, die überschüssige Salpetersäure abgeraucht, $20 \mathrm{ccm}$ Schwefelsäure zugefügt und eingedampft, bis Schwefelsäuredümpfe auftreten. Der Rückstand wird mit krystallisirtem Ferrosulfat und reiner Salzsäure versetzt und destillirt. Das Destillat wird in Wasser aufgefangen, das Arsen daraus mittelst Schwefelwasserstoff- gefällt. Das Arsentrisulfid wird mit Wasser, Alkohol und Schwefelkohlenstoff aurgewaschen und in Ammoniak gelöst. Die ammoniakalische Lösung wird zur Trockne gedampft und bei $100^{\circ}$ bis zur Gewichtskon$\therefore \tan z$ getrocknet.

G. Sonntag.

Brunton, Stevenson, Salamon, Luff, Buckley, Fletscher und Moulton: Berich t der Kommission über Arsenikim Bier an die Central-Vereinigung der Brauer von Manchester. - Analyst 1901, 26, 13-15.

Nach vorgenommener Untersuchung der Zucker und aller in den Brauereien Manchesters verwendeten Materialien (nit Ausnahme des Malzes) kam die Kommission zu der Ueberzeugung, dass die in den Bieren gefundenen schädlichen Mengen Arsen vom Zucker der Firma Bostock \& Cie. in Liverpool herstammten, welche in ihrem Etablissement unreine Schwefelsäure verwendete. Die Kommission hatte die Entfernung alles mit solchem Zucker hergestellten Bieres angeordnet und somit jede Gefahr beseitigt. Bei der grossen Tragweite dieser Angelegenheit hat die Kommission auch alle anderen im Lande zur in Verwendung gekommenen Zuckerprodukte untersucht und arsenfrei befunden. In einzelnen Proben der untersuchten Malze und Hopfen wurden gleichwohl auch Spuren von Arsen nachgewiesen, welchen man aber die schädliche Wirkung der Biere keineswegs zuzuschreiben habe. Zum Schutze der Bierkonsumenten sollen fortan die Biere nach einem vorgeschlagenen Verfahren auf Arsen geprüft werden. Man bringt $200 \mathrm{ccm}$ Bier in eine Porcellan-Abdampfschale, erhitzt zum Kochen setzt $30 \mathrm{ccm}$ reiøe koncentrirte Salzsäure zu und ein Stückchen reines blankes Kupferblech von $1 / 4$ bis $1 / 2$ Zoll Breite. Die Flüssigkeit wird 45 Minuten kochend erhalten. Wenn nach dieser Zeit das Kupfer glänzend roth erscheint, so ist das Bier frei von Arsenik. Hat sich auf dem Kupfer ein Niederschlag ab- 
gesetzt, dann ist das Kupferblech nacheinander mit Wasser, Alkohol und Aether abzuwaschen bei $100^{\circ} \mathrm{C}$. zu trocknen und zur Sublimation in ein enges Reduktionsröhrchen (nicht über 2 Zoll lang) zu bringen. Znr Reduktion bedient man sich einer Spiritusflamme.

L. Aubry.

E. Prior: Zur biologischen Bierprüfung. - Bayerisches Braner-Journal 1901, 11, 121.

Es gelangen nicht selten in den gährungsphysiologischen Laboratorien der Versuchsstationen Biere zur Untersuchung, welche neben Hefen der verschiedensten Art auch Mikroorganismen enthalten, die in steriler Würze bei uubeschränktem Luftzutritt vornehmlich an der Oberfläche der Nährflüssigkeit wachsen und die anwesenden Hefen derart überwuchern, dass es unmöglich ist, diese rein zu kultiviren und zu charakterisiren. $\mathrm{Zu}$ diesen hier in Betracht kommenden Organismen gehören die Mykoderma-Arten und Säurebakterien. Nun kann man zwar durch Zusätze zur Nährflüssigkeit die Entwickelung der angeführten Organismen hemmen, ohne diejenige der Hefen bedeutend zu beeinflussen, doch hat Verf. gefunden, dass der Erfolg nicht in allen Fällen sicher ist und gewöhnliche Hefearten durch solche Zusätze ebenfalls in der Entwickelung zurückgehalten werden.

Diese Nachtheile lassen sich in der Regel vermeiden, wenn man die Nährlösung, Bierwürze etc., in welcher die Bierabsätze kultivirt werden, mit einem in strömenden Wasserdampf zuvor sterilisirten Vaselinöl $2-3 \mathrm{~mm}$ hoch nach der Aussaat der Zellen überschichtet. Diese vom Verf. in den gährungsphysiologischen Laboratorien der Versuchsstation für Bierbrauerei in Nürnberg seit Monaten angewandte, sehr einfache Methode hat in den meisten Fällen sehr gute Dienste geleistet, indem es bei Anwesenheit der erwähnten Mikroorganismen immer gelungen ist, die vorhandenen Hefen vor Ueberwucherung zu schützen und zur Entwickelung zu bringen, um sie alsdann nach den Methoden von $\mathrm{Hansen}$ weiter kultiviren und analysiren zu können. Es werden stets zwei Kulturen gemacht, eine mit und eine ohne Vaselinölschicht. Auch zur Trennung anderer aërober und anaërober Mikroorganismen dürfte die beschriebene einfache Methode, wenn Beschränkung des Luftzutritts genügt, gute Dienste leisten und die bekannten meist umständlichen Verfahren ersetzen können.

\section{j. Brand.}

Hantke und Kremer: Bestimmung des Mousseux und des Schaumstandes von Bier in Zahlen. - Letters on Brewing Hantke's Brewers School and Laborat., Milwaukee Juli 1900, 75-81.

Die Verff. versuchten eine praktische, leicht ausführbare Methode aufzufinden, mittelst welcher es möglich wäre, den Trieb und den Schaumstand von Bieren vergleichsweise mit anerkannt guten, in dieser Beziehung befriedigenden Bieren festzustellen.

Zu diesem Zweck wurde zuerst die Gesammt-Kohlensäure bestimmt. Apparat und Methode, bei welcher die Kohlensäure in mit Ammoniak versetzter Chlorbaryumlösung deren Gehalt an Baryum gewichtsanalytisch festgestellt worden war, eingeleitet wurde, werden beschrieben. Ferner wird der Kohlensäuregehalt verschiedener Biere nach längerem offenen Stehen bei verschiedenen Temperaturen, sowie der Druck der Kohlensäure durch ein Quecksilbermanometer bestimmt. Aus der Arbeit lassen sich folgende Schlüsse ziehen:

1. Bier absorbirt selbst bei $20^{\circ} \mathrm{C}$. mehr Kohlensäure, als das im Bier enthaltene 
Alkohol-Wassergemisch nach Berechnung enthalten kann. Dies bestätigt nur die Resultate, welche Langer und Schulze bereits im Jahre 1883 gefunden haben.

2. Man kann die Menge der durch Berechnung gefundenen absorbirten Kohlensäure nur als Minimalgrenze betrachten und in Folge dessen Biere als ungenügend bezeichnen, wenn weniger absorbirte Kohlensäure gefunden wird.

3. Gute Biere lassen mindestens $0,19 \%$, meist aber über $0,2 \%$ absorbirter Kohlensäure nach 24-stündigem Stehen bei $20^{\circ} \mathrm{C}$. nachweisen.

4. Gute Biere besitzen bei $20^{\circ} \mathrm{C}$. einen Druck von über 14 Pfund. Daher kamm man durch die Druckbestimmung schon einen Anhalt für die Güte in Bezug auf Schaumbaltigkeit erhalten, doch wird der Druck wohl selten über $15^{1 / 2}$ Pfund gehen, demn ein höherer Druck lässt vielleicht auf Ueberspundung schliessen.

5. Druckbestimmung in Verbindung mit Feststellung der absorbirten Kohlensäure mittelst Barythydrat und Titration mit Schwefelsäure dürften praktisch einen genügenden Anhaltspunkt für gutes Mousseux und Schaumstand geben, namentlich wenn durch die Analyse die sonstigen Eigenschaften des Bieres bekannt sind.

J. Brand.

Dunbar unter Mitwirkung von K. Farnsteiner, K. Lendrich und J. Zink: Ueber Bieruntersuchung. - 3. Bericht des hygienischen Instituts Hamburg, 1898/99. Hamburg 1900, 96-101.

Wie aus den angeführten Tabellen hervorgeht, zeigen die nach Torno ë gefundenen Extraktwerthe genügend gute Uebereinstimmung. Bei den vergleichenden Alkoholbestimmungen konnte jedoch, entgegen der Ansicht von Prior und anderen Forschern eine genügende Uebereinstimmung nicht beobachtet werden. Die Abweichungen sincl in der Mehrzahl so erheblich, dass sie auch für die Praxis als viel zu gross bezeichnet werden müssen.

C. Mai.

Adolf Beythien: Ueber saccharinhaltige sog. "süsse Weizenmalzextrakte“. - Zeitschr. angew. Chem. 1901, 14, 247-254.

Im Verlaufe der während des Jahres 1899 im chemischen Untersuchungsamte der Stadt Dresden vorgenommenen Untersuchung von Bierproben gelangten auch mehrere Proben zur Einlieferung, welche in kleineren Geschäften zum Preise von 7-10 Pf. für die 1/2 l-Flasche feilgeboten worden waren und von den revidirenden Beamten, in der Meinung Weizenmalzbier vor sich zu haben, entuommen worden waren. Die Proben waren von dunkler Farbe, zeigten beim Oeffnen der Flaschen lebhafte Kohlensäure-Entwickelung, und hatten denselben Geschmack wie versüsstes einfaches Bier. Auch die chemische Analyse stimmte damit überein; sie zeigte 1,32,2\% Alkohol, 2,7-3,5\% Extrakt entsprechend einem Stammwürzegehalt von $\check{5}, 2-7,9 \%$. Auserdem war Saccharin zugegen. Der mittlere Gehalt der Dresdener einfachen Biere (Mittel aus 42 Analysen) ist für Alkohol 1,24\%, für Extrakt 3,34\%. Auf Grund diewer Uebereinstimmung wurden die Produkte als mit Saccharin versüsste einfache Biere angesehen und ihre Herstellung und Freihaltung als strafbar im Sinne des Gesetzes betr. den Verkehr. mit künstlichen Süssstoffen bezeichnet. Nun stellte sich heraus, dass ein gewisser Schüller einen sog. hochkoncentrirten süssen Weizenmalzextrakt herstellte und verkaufte, der zur Anfertigung dieses Bieres nach dessen Angaben in der Weise benutzt wurde, dass man $201 \mathrm{Kulmbacher} \mathrm{Bier} \mathrm{mit} 79 \mathrm{l}$ Wasser, $1^{1 / 2} \mathrm{~kg}$ Zucker und $1 / 21$ des saccharinhaltigen Extraktes vermischte. Der Verfertiger behauptete ein solches Getränke sei nicht Bier, sondern ein Bier ähnliches Getränk, für welches der Saccharinzusatz unter Deklaration zulässig sei. 
Die Untersuchung des koncentrirten Extraktes, eines schwarzbraunen dickflüssigen Syrups von stark süssem Geschmacke, ergab folgende procentige Zusammensetzung: Extrakt 67,89, Asche 1,38, Eiweissstoffe 1,74, Zucker (Maltose) 18,22, Säure 1,44, Saccharin 0,21. Dasselbe war als der Hauptsache nach als eine saccharinhalige Zuckercouleur anzusehen, welche im Hinblick auf den minimalen Eiweissgehalt nur Spuren Malz enthalten konnte.

Die Gerichte legten nun 5 Sachverständigen folgende Fragen vor: 1. Sind die Getränke Bier oder bierähnliche Getränke? Filsinger, Dreverh off und Renk erklärten das nach den Schüller'schen Vorschriften erzeugte Produkt nicht als Bier, sondern als bierähnliches Getränk, während der Verfasser und Drenkmann dasselbe als ein Bier nach Art des sog. Weizenbieres bezeichneten, und die Existenz von bierähnlichen Getränken bezw. das Vorhandensein eines Handelsbegriffs „bierähnlicher Getränke“ bestritten. Die Gerichte haben sich dem Gutachten der beiden letzten Sachverständigen nicht angeschlossen, sondern entschieden, dass die vorliegenden Gemische von Bier, Wasser und saccharinhaltigen Extrakten nicht Bier, sondern bierähnliche Getränke darstellen, zu deren Herstellung die Verwendung von Saccharin zulässig sei. Diese Entscheidung erscheint für die Wirksamkeit des Gesetzes betr. den Verkehr mit künstlichen Süssstoffen nicht unbedenklich, indem sie durch Einführung des bislang unbekannten Begriffes „bierähnliche Getränke" eine in dem Gesetze nicht vorhandene Lücke künstlich konstruirt, die dazu führen muss, das ganze Gesetz illusorisch zu machen.

J. Brand.

Mecke: Methon. - Zeitschr. öffentl. Chem. 1901, 7, 241-242.

Von der Firma Franz Hermann Loebel-Dresden wird mit grosser Reklame unter der Bezeichnung Methon eine Essenz, bezw. ein daraus hergestelltes Getrünk als „flüsssiges Brot", „alkoholfreies Bier" in den Handel gebracht. $1 \mathrm{~kg}$ der Essenz soll unter Zusatz von Zucker und Weinsäure zur Bereitung von 250 Flaschen Getränk dienen. Verf. hat weder Hopfen noch Malz nachweisen können. Da das Getränk weder Phosphorsäure noch Stickstoff enthielt, so scheint die Essenz nichts weiter zu sein, als eine parfümirte, mit Schaumessenz versetzte Zuckerkcouleur. G. Sonntag.

Johann J. Vanha: Vegetationsversuche über den Einfluss der einzelnen Nährstoffe auf die Gestaltung und Abänderung der Wertheigenschaften der Gerste. - Zeitschr. landw. Versuchsw. Oesterreich 1901, 4, 40-70.

Johann J. Vañha: Vegetationsversuche über den Einfluss verschiedener mechanischer Zusammensetzung desselben Bodens auf die Gerstenpflanze. - Zeitschr. landw. Versuchsw. Oesterreich 1901, 4, 99-114.

Bohuslav Procházka: Studien über die böhmische Gerste. - Zeitschr. landw. Versuchw. Oesterreich 1901, 4, 81-95.

A. Lang: Gersten der Ernte 1900. - Zeitschr. ges. Brauw. 1901, 94, 521.

H. Hanow: Die im April, Mai, Juni, Juli und August d. J. untersuchten Malze. - Wochenschr. Brauerei 1901, 18, 185, 317, 389-390 und 445.

O. Reinke: Ueber Verbesserungen und Untersuchungen der Mälzerei- und Brauereiprodukte im Lichte der neuesten Forschungen, insbesondere a uch über Enzyme. - Wochenschr. Brauerei 1901, 18, 372-375. 
Ueber die Verwendung des ,Neigebieres" beim Bierausschanke. Gutachten des k. k. Obersten Sanitätsrathes in Wien. - Oesterr. Sanitätswesen 1901, 13, 393 bis 395. Vergl. den Abschnitt "Bier" auf Seite 1184 unter "Gesetze u. s. w.“

\section{Gesetze, Gesetz - Entwïrfe, Verordnungen u. s. w., Gerichts - Entscheidungen.}

\section{Milch und Käse.}

Sachsen. Bekanntmachungen des Raths der Stadt Dresden, den Verkehr mit Milch betr. Vom 31. Juli 1900 und 26. Februar 1901. - Veröffentl. Kaiserl. Gesundh. 1901, 25, $576-578$.

Kuhmilch darf nur in den Verkehr gebracht werden als Vollmilch in zwei Sorten, nämlich mit mindestens und mit weniger als $2,8 \%$ Fettgehalt, und als abgerahmte, d. h. auch nur theilweise abgerahmte einschliesslich sog. Halbmilch. Abgekochte sterilisirte oder pasteurisirte Milch ist als solche zu bezeichnen ( $\$ 1$ ). Anders als durch Abrahmen, Abkochen, Sterilisiren, Pasteurisiren oder Gefrieren veränderte, besonders auch mit Wasser oder Konservirungsmitteln versehene Milch ist unzulässig ( $\$ 2$ ). An den Milchgefässen, bei geschlossenen Wagen über den Abflusshähnen, sind entsprechende Bezeichnungen fest anzubringen (\$3). Milch von kranken Thieren, aus Gehöften oder Orten, in denen Typhus- oder Cholera-Epidemien beobachtet sind, wenige Tage vor und bis zum 6. Tage nach dem Abkalben abgemolkene, bittere, schleimige, aussergewöhnlich gefärbte, mehr wie 19 Säuregrade aufweisende, verdorbene oder sonst Ekel erregende Milch ist ausgeschlossen. Marktmilch darf nicht mehr als $8 \mathrm{mg}$ Milchschmutz pro Liter enthalten ( $\S 4)$. Alle Verkehrsmilch ist auf Erfordern der Beamten der Wohlfahrtspolizei zur Untersuchung und Prïfung bereit zu stellen (\$5). Beförderung, Aufbewahrung und Verarbeitung der Milch müssen so geschehen, dass dadurch deren Geniessbarkeit und Haltbarkeit nicht beeinträchtigt wird. An ansteckenden oder Ekel erregenden Krankheiten leidende, mit Geschwüren oder Ausschlägen an Händen oder im Gesicht behaftete oder mit derartig Erkrankten in unmittelbare Berührung kommende Personen dürfen sich mit dern Milchvertriebe nicht beschäftigen (\$6). Als Kur-, Kinder-, Kontrolle-, Gesundheitsmilch oder ähnlich bezeichnete Milch muss böheren Ansprüchen an Gewinnung, Aufbewahrung und Beförderung genügen. Der Verkauf solcher ebenso wie sterilisirter oder pasteurisirter Milch muss behördlich genehmigt sein (\$7). Auch nach Ertheilung der Genehmigung sind gewisse Vorschriften über die Einstellung neuer Thiere, über die thierärztliche Ueberwachung der Thiere, deren Fütterung und Verpflegung, das Verhalten bei Erkrankungen, das Pflegepersonal, das Melken, die alsdann folgende Behandlung der Milch und eine ärztliche Ueberwachung des Gesammtbetriebes zu beobachten (\$8). Andere Milcharten, sowie Rahm, Schlicker-, Buttermileh und Molken sind nach $\S 3$ als solche zu bezeichnen und unterliegen den Bestimmungen der $\$ \S 6$ und 7 . Rahm muss mindestens $10 \%$ Fettgehalt haben $(\$ 9)$.

\section{Mehl- und Backwaaren.}

Deutsches Reich. Rechtsprechung. Urtheil des Reichsgerichts, betr. mit Stecknadeln versehene Semmeln. Vom 11. November 1898. - Veröffentl. Kaiserl. Gesundh. Beil. 1901, 5, 239*-240*.

Die Dienstmagd eines Bäckers hatte wiederholt in die zur Ablieferung bestimmten Semmeln Stecknadeln derartig gesteckt, dass sie von aussen nicht sichtbar waren, aber beim Entzweibrechen der Semmeln jedesmal entdeckt wurden. Auf Fälle solcher Art sei § 12 N.M.G. nicht anwendbar. Vielmehr sei nach dem Geiste dieses Gesetzes, das zwar für die Anwendung seiner Strafbestimmungen im Einzelfalle gemeingefährliches Handeln nicht erheische, dennoch 